\title{
FLORÍSTICA E ESTRUTURA DA COMUNIDADE ARBÓREA DE UMA FLORESTA ESTACIONAL SEMIDECIDUAL SECUNDÁRIA EM VIÇOSA, MG ${ }^{1}$
}

\author{
Antonio Jorge Tourinho Braga², Eduardo Euclydes de Lima e Borges ${ }^{3}$ e Sebastião Venâncio Martins ${ }^{3}$
}

\begin{abstract}
RESUMO - Este trabalho teve como objetivo estudar a composição florística e a estrutura da comunidade arbórea, bem como a distribuição das espécies em diferentes grupos ecológicos e os solos dos estádios inicial e avançado, de uma Floresta Estacional Semidecidual localizada no campus da Universidade Federal de Viçosa,

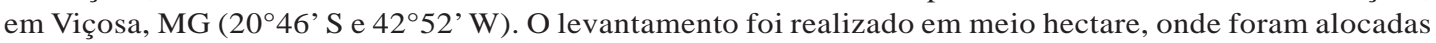
10 parcelas de 25 x 10 m em cada trecho, sendo amostrados todos os indivíduos com circunferência do tronco a 1,30 m do solo (CAP) $\geq 15 \mathrm{~cm}$. Registraram-se 820 indivíduos, sendo 440 no trecho de floresta inicial e 380 no de floresta avançada. No levantamento florístico da floresta inicial foram amostradas 76 espécies, pertencentes a 28 famílias, destacando-se como as de maior número de indivíduos Fabaceae (137), Urticaceae (45) e Sapindaceae (41) e, as com os maiores valores de importância, Cecropia glaziovii Snethl., Anadenanthera peregrina (L.) Speng., Piptadenia gonoacantha (Mart.) Macbr., Allophylus sericeus Radlk., Siparuna guianensis Aubl. e Maclura tinctoria (L.) Don ex Steud. O índice de diversidade de Shannon (H’) foi de 3,57 nat.ind. ${ }^{-1} \mathrm{e}$ a equabilidade (J'), de 0,82. Já na floresta avançada foram amostradas 59 espécies, distribuídas em 26 famílias, das quais Fabaceae (103), Meliaceae (49) e Flacourtiaceae (34) sobressaíram com maior número de indivíduos. Por sua vez, as espécies com os maiores valores de importância foram A. peregrina, Trichilia pallida Swartz, Cariniana estrellensis (Raddi) Kuntze., P. gonoacantha, Rollinia silvatica Mart. e S. guianensi. O índice de diversidade de Shannon (H') foi de 3,49 nat.ind. ${ }^{-1}$ e a equabilidade (J'), de 0,85. No contínuo, o índice de Shannon (H') e a equabilidade (J') foram de 3,82 nat.ind. ${ }^{-1}$ e 0,84 , respectivamente. Cada floresta apresentou distinta identidade florística e estrutural, tendendo a ser minimizada com o avanço da sucessão.
\end{abstract}

Palavras-chave: Fitossociologia, Estádios sucessionais e Grupos ecológicos.

\section{FLORISTIC AND STRUCTURE OF TREE COMMUNITY IN SECONDARY SEMIDECIDUOUS SEASONAL FOREST IN VIÇOSA, MG}

\begin{abstract}
The objective of this work was to study the floristic and structure of a tree community as well as the distribution of the species in different ecological groups and the soil at the initial and advanced stages of a semideciduous seasonal forest located in the campus of Universidade Federal de Viçosa in Viçosa, MG ( $20^{\circ} 46^{\prime} S$ and $42^{\circ} 52^{\prime} \mathrm{W}$ ). The survey was carried out in a half hectare where ten $25 \times 20$ - $m$ plots were allocated in each stretch, being sampled all individuals with circumference at breast height at $1.30 \mathrm{~m}$ from the soil (DCH) $\geq 15 \mathrm{~cm}$. It was registered 820 individuals, 440 in the initial stretch of the forest and 380 in the advanced stretch of the forest. In the floristic survey of the initial forest, it was sampled 76 species which belonged to 28 families, standing out as the one with the greatest number of individual the following: Fabaceae (137), Urticaceae (45) and Sapindaceae (41), and the species with the greatest importance value were the following: Cecropia glaziovii Snethl.,Anadenanthera peregrina (L.) Speng., Piptadenia gonoacantha (Mart.) Macbr., Allophylus sericeus Radlk., Siparuna guianensis Aubl. and Maclura tinctoria (L.) Don ex Steud. The Shannon diversity index ( $\left.H^{\prime}\right)$ was 3.57 nat.ind.$^{-1}$ and the Pielou evenness ( $\left.J^{\prime}\right)$ was 0.82 . In the advanced forest it was sampled 59 species distributed among 26 families, from which Fabaceae (103), Meliaceae (49) and Flacourtiaceae (34),stood out with the greatest number of individuals. In turn, the species with the highest importance value were A. peregrina, Trichilia pallida Swartz, Cariniana estrellensis (Raddi) Kuntze, P. gonoacantha, Rollinia silvatica Mart. and S. guianensi. Shannon diversity index ( $\left.H^{\prime}\right)$ was 3.49 nat.ind. ${ }^{-1}$ and the Pielou evenness ( $\left.J^{\prime}\right)$ was 0.85. In the continuum, Shannon diversity index ( $\left.H^{\prime}\right)$ was 3.82 nat.ind. ${ }^{-1}$ and the Pielou evenness ( $\left.J^{\prime}\right)$ was 0.84. Each forest presented distinct floristic and structural idendity, tending to be minimized succession advanced.
\end{abstract}

Keywords: Phytossociology, Successional stages and Ecologic group.

\footnotetext{
${ }^{1}$ Recebido em 09.10.2009 e aceito para publicação em 14.04.2011.

${ }^{2}$ Instituto Federal de Educação, Ciência e Tecnologia Baiano, IFBAIANO, Brasil. E-mail: < antonio.braga@catu.ifbaiano.edu.br>.

${ }^{3}$ Universidade Federal de Viçosa, UFV, Brasil. E-mail: <elborges@ufv.br> e < venancio@ufv.br>.
} 


\section{INTRODUÇÃO}

A área de Floresta Atlântica no Estado de Minas Gerais compreende diferentes formações florestais que, na sua maior parte, é ocupada por Florestas Estacionais Semideciduais, as quais recobrem todo o leste, com maiores extensões nas porções sudeste e sul, estendendo-se, ainda, pelos vales dos rios Paranaíba, Grande e seus afluentes, e nos encraves de araucária localizados no sul do Estado (COSTA et al., 1998).

Na Zona da Mata mineira, o ciclo do café seguido da pecuária e, mais recentemente, do plantio de canade-açúcar, modificaram severamente as Florestas Estacionais Semideciduais da região (MEIRA-NETO; SILVA, 1995). Esses processos de perturbação e de fragmentação da vegetação, semelhantes aos ocorridos nos demais ecossistemas brasileiros, demonstram a necessidade do conhecimento e do entendimento da complexa dinâmica que envolve os poucos remanescentes florestais nativos. O conhecimento da identidade das espécies e o seu comportamento em comunidades vegetais, que se iniciam pelo levantamento da florística, são o começo de todo processo de compreensão desse ecossistema (MARANGON et al., 2003).

Estudos sobre a composição florística e a ecologia das comunidades vegetais são, desse modo, fundamentais para embasar quaisquer iniciativas de preservação e conservação de remanescentes florestais (OLIVEIRA-FILHO et al., 1994), bem como para orientar medidas de manejo a serem adotadas, a partir do entendimento do processo de dinâmica sucessional instaurada nos diferentes estádios sucessionais de uma comunidade florestal (PINTO, 2005).

Considerando o exposto, este estudo foi desenvolvido com o objetivo de conhecer a composição florística e estrutural da comunidade arbórea, bem como a distribuição das espécies em diferentes grupos ecológicos, e os solos somente para caracterizar o ambiente dos estádios inicial e avançado de uma Floresta Estacional Semidecidual, situada no Campus da Universidade Federal de Viçosa.

\section{MATERIAL E MÉTODOS}

O estudo foi conduzido no fragmento florestal denominado Mata da Agronomia, que compreende, aproximadamente, 50 ha, localizado no Campus da
Universidade Federal de Viçosa, no Município de Viçosa, MG, nas coordenadas de $20^{\circ} 46^{\prime}$ S e $42^{\circ} 52^{\prime}$ W , a uma altitude que varia entre 688 e $782 \mathrm{~m}$.

A Mata da Agronomia é caracterizada como Floresta Estacional Semidecidual Montana (VELOSO et al., 1991). A Mata possui histórico de mais de 50 anos de regeneração natural, instaurada após a cobertura vegetal original ter sido removida por corte raso e substituída por plantio sequencial de café e pastagem. Um dos trechos escolhidos, denominado neste estudo floresta inicial, encontrava-se em processo de regeneração substituindo a pastagem há aproximadamente 28 anos. Nesse trecho, situando-se bem às margens do aceiro que fazia divisa com o campo experimental Prof. Diogo Alves Melo (2046.105'S e $42^{\circ} 52.061^{\prime} \mathrm{W}$ ), a altitude variava entre 688 e $737 \mathrm{~m}$. Outro trecho de floresta com mais de 50 anos foi denominado floresta avançada e encontrava-se em estádio já avançado de sucessão, adentrando a Mata (2046.197's e $42^{\circ} 52.061^{\prime} \mathrm{W}$ ), com atitude entre 708 e $782 \mathrm{~m}$.

A topografia local é acidentada, com vales estreitos e úmidos, com predominância de duas classes de solo, estando o Latossolo Vermelho-Amarelo álico no topo das elevações e encostas, enquanto o Argissolo VermelhoAmarelo Câmbico dominava as feições do relevo denominadas terraços fluviais (RESENDE et al., 1988). O clima da região, através do sistema de Köppen, é do tipo Cwb, mesotérmico com verões chuvosos e invernos frios e secos. A temperatura média anual é de $19^{\circ} \mathrm{C}$ e a umidade relativa do ar varia em torno de $80 \%$, sendo a precipitação média anual de $1.314 \mathrm{~mm}$ (CASTRO et al., 1983).

Os estudos florístico e fitossociológico foram realizados em 10 parcelas permanentes, alocadas em cada trecho da floresta, de acordo com o método de parcelas (MUELLER-DOMBOIS; ELLENBERG, 1974), medindo cada uma $25 \times 10 \mathrm{~m}$, alocadas em intervalos de $5 \mathrm{~m}$, totalizando com os dois trechos uma área amostral de 0,5 ha.

Todos os indivíduos arbóreos, com circunferência a 1,30 m do solo (CAP) igual ou superior a $15 \mathrm{~cm}$, foram amostrados. No caso dos indivíduos perfilhados, foram considerados aqueles que apresentassem pelo menos um perfilho com CAP $\geq 15 \mathrm{~cm}$. Foram registrados os valores de CAP, estimada a altura e coletado material botânico para identificação de cada indivíduo amostrado. Os indivíduos mortos em pé também foram contabilizados e medidos, sendo classificados como "mortas". 
O material botânico foi identificado por meio de consulta ao Herbário do Departamento de Biologia Vegetal (VIC) da Universidade Federal de Viçosa, e as espécies foram classificadas em famílias de acordo com o sistema Angiosperm Phylogeny Group II (APG II, 2003). A confirmação dos nomes das espécies e de seus autores foi obtida através de consultas ao site Missouri Botanical Garden (http://www.mobot.org), bem como através de literaturas especializadas.

Os dados foram analisados com o auxílio do Programa Mata Nativa 2 (CIENTEC, 2006), que calculou o índice de diversidade de Shannon (H'), o índice equabilidade de Pielou (J') (BROWER; ZAR, 1984) e os parâmetros fitossociológicos usuais (MUELLERDOMBOIS; ELENBERG, 1974).

As espécies amostradas foram classificadas em categorias sucessionais, conforme os critérios adotados por Gandolfi et al. (1995), além de classificações adotadas nos estudos de Martins e Rodrigues (2002) e Martins et al. (2002, 2004). Os taxa com informações divergentes ou ausentes foram classificados como "não classificado".

As amostras de solo foram coletadas de forma aleatória em cada parcela, com o auxílio de um trado holandês na camada de 0 a $10 \mathrm{~cm}$, totalizando 20 amostras coletadas, cada uma composta de 10 subamostras. Após a coleta, as amostras foram submetidas às análises química (rotina e matéria orgânica) e física no Laboratório do Departamento de Solos da Universidade Federal de Viçosa. As variáveis edáficas obtidas nas florestas inicial e avançada foram comparadas pelo teste t, de Student.

\section{RESULTADOS}

Foram identificados, nas duas áreas de floresta amostradas, 820 indivíduos de porte arbóreo, correspondentes a 96 espécies, distribuídas em 76 gêneros pertencentes a 35 famílias, sendo seis espécies identificadas apenas em nível de gênero e três em nível de família. Ocorreram também 39 espécies comuns nos dois fragmentos. Entre as espécies exclusivas de cada área, as florestas inicial e avançada apresentaram 37 e 20 espécies, respectivamente.

Na floresta inicial foram amostrados 440 indivíduos, distribuídos em 76 espécies, pertencentes a 28 famílias. A densidade total por hectare e a área basal estimadas nesse trecho de floresta foram, respectivamente, de 1.760 indivíduos e 20,01 m².ha-1, cujo maior diâmetro, de $140 \mathrm{~cm}$, foi apresentado por um indivíduo de Eucalyptus sp., remanescente de plantio que havia na área antes do processo de regeneração (Tabela 1). Já na floresta avançada foram amostrados 380 indivíduos, sendo 59 espécies pertencentes a 26 famílias. Nesse trecho de floresta foram estimadas a densidade total de 1.520 indivíduos. ha ${ }^{-1}$ e uma área basal de 22,96 m² ha-1, sendo o maior diâmetro, de $222 \mathrm{~cm}$, obtido de um indivíduo de Cariniana estrellensis (Tabela 2).

As espécies de maior valor de importância (VI), na floresta inicial, foram C. glaziovii, Anadenanthera peregrina, Piptadenia gonoacantha, Allophylus sericeus, Siparuna guianensis e Maclura tinctoria. Essas seis espécies totalizaram 41,88\% dos indivíduos amostrados, sendo o restante distribuído entre as demais 70 espécies. Já na floresta avançada as espécies que apresentaram maior importância foram A. peregrina, T. palida, $C$. estrellensis, $P$. gonoacantha, Rollinia silvatica e S. guianensis, compondo 37,14\% do valor de importância. Os indivíduos mortos, no total de 23, ocuparam a terceira posição na estrutura da comunidade arbórea da floresta avançada, correspondendo a 5,83\% do total amostrado, com frequência relativa de $4,5 \%$, o que demonstra a boa distribuição desses indivíduos por toda a amostra e o avanço do estádio de sucessão dessa área.

Entre as duas espécies que figuraram como as mais importantes nos trechos de florestas estudados, A. peregrina apresentou tendência a agrupamento, com índice de Payandeh ( $\mathrm{Pi}$ ) de 1,37 para o conjunto, aparecendo em 19 das 20 parcelas estudadas, enquanto C. glaziovii, espécie colonizadora de clareiras e bordas em matas secundárias e capoeiras, se concentrou na floresta inicial, em sete das 10 parcelas alocadas de forma agrupada $(\mathrm{Pi}=4,48)$.

Das 35 famílias amostradas nas duas áreas pesquisadas, 19 foram comuns aos dois trechos de florestas. Entre as famílias exclusivas de cada floresta, nove foram amostradas apenas na inicial (Achariaceae, Asteraceae, Cannabaceae, Lacistemataceae, Lamiaceae, Melastomataceae, Ochnaceae, Urticaceae e Verbenaceae), enquanto na avançada sete famílias são exclusivas: Arecaceae, Celestraceae, Combretaceae, Icacinaceae, Nyctaginaceae, Rosaceae e Vochysiaceae.

Entre as famílias que apresentaram maior número de espécies na floresta inicial estão Fabaceae (18), Euphorbiaceae (6) e Meliaceae (5). Já Fabaceae (137), Urticaceae (45) e Sapindaceae (41) se destacaram com o maior número de indivíduos.

Revista Árvore, Viçosa-MG, v.35, n.3, p.493-503, 2011 
Tabela 1 - Espécies arbóreas amostradas no trecho de floresta inicial da Mata da Agronomia, em Viçosa, MG, com os respectivos números de indivíduos $(\mathrm{N})$ e parâmetros fitossociológicos: frequência absoluta (FA), frequência relativa (FR), densidade absoluta (DA), densidade relativa (DR), dominância absoluta (DoA), dominância relativa (DoR), valor de cobertura (VC) e valor de importância (VI), em ordem decrescente de VI.

Table 1 - Tree species sampled in initial forest in Mata da Agronomia, in Viçosa/MG, with their respective number of individuals (N) and phytosociological parameters: absolute frequency (FA), relative frequency (FR), absolute density (DA), relative density (DR), absolute dominance (DoA), relative dominance (DoR), cover value (VC), importance value (VI\%), in decreased order of VI.

\begin{tabular}{|c|c|c|c|c|c|c|c|c|c|}
\hline Nome Científico & $\mathrm{N}$ & DA & DR & FA & FR & DoA & DoR & VC (\%) & VI (\%) \\
\hline$\overline{\text { Cecropia glaziovii Snethl. }}$ & 44 & 176 & 10 & 70 & 3,54 & 5,6 & 27,94 & 18,97 & 13,83 \\
\hline Anadenanthera peregrina Speg. & 50 & 200 & 11,36 & 100 & 5,05 & 2,085 & 10,4 & 10,88 & 8,94 \\
\hline Piptadenia gonoacantha Macbride & 36 & 144 & 8,18 & 90 & 4,55 & 1,801 & 8,99 & 8,58 & 7,24 \\
\hline Allophylus sericeus Radlk. & 38 & 152 & 8,64 & 70 & 3,54 & 0,844 & 4,21 & 6,42 & 5,46 \\
\hline Siparuna guianensis Aubl. & 29 & 116 & 6,59 & 30 & 1,52 & 0,334 & 1,67 & 4,13 & 3,26 \\
\hline Maclura tinctoria (L.) D. Don ex Steud. & 13 & 52 & 2,95 & 80 & 4,04 & 0,49 & 2,44 & 2,7 & 3,15 \\
\hline Rollinia sp. & 12 & 48 & 2,73 & 50 & 2,53 & 0,622 & 3,1 & 2,91 & 2,78 \\
\hline Apuleia leiocarpa Macbride & 12 & 48 & 2,73 & 40 & 2,02 & 0,655 & 3,27 & 3 & 2,67 \\
\hline Morta & 11 & 44 & 2,5 & 60 & 3,03 & 0,482 & 2,41 & 2,45 & 2,65 \\
\hline Luehea grandiflora Mart. \& Zucc. & 9 & 36 & 2,05 & 50 & 2,53 & 0,245 & 1,22 & 1,64 & 1,93 \\
\hline Protium warmingianum March. L. & 9 & 36 & 2,05 & 60 & 3,03 & 0,137 & 0,68 & 1,37 & 1,92 \\
\hline Albizia polycephala (Benth.) Killip & 7 & 28 & 1,59 & 40 & 2,02 & 0,393 & 1,96 & 1,77 & 1,86 \\
\hline Croton urucurana Baill. & 6 & 24 & 1,36 & 30 & 1,52 & 0,528 & 2,63 & 2 & 1,84 \\
\hline Rollinia silvatica Mart. & 6 & 24 & 1,36 & 50 & 2,53 & 0,32 & 1,6 & 1,48 & 1,83 \\
\hline Ocotea laxa Mez & 8 & 32 & 1,82 & 30 & 1,52 & 0,42 & 2,1 & 1,96 & 1,81 \\
\hline Copaifera langsdorffii Desf. & 6 & 24 & 1,36 & 50 & 2,53 & 0,179 & 0,89 & 1,13 & 1,59 \\
\hline Sapium glandulatum (Vell.) Pax. & 8 & 32 & 1,82 & 30 & 1,52 & 0,202 & 1,01 & 1,41 & 1,45 \\
\hline Casearia arborea (Rich.) Urb. & 8 & 32 & 1,82 & 30 & 1,52 & 0,188 & 0,94 & 1,38 & 1,42 \\
\hline Zanthoxylum rhoifolium Lam. & 5 & 20 & 1,14 & 40 & 2,02 & 0,175 & 0,87 & 1,01 & 1,34 \\
\hline Dalbergia nigra Allem. Ex Benth. & 4 & 16 & 0,91 & 40 & 2,02 & 0,211 & 1,05 & 0,98 & 1,33 \\
\hline Eucalyptus sp. & 1 & 4 & 0,23 & 10 & 0,51 & 0,624 & 3,11 & 1,67 & 1,28 \\
\hline Astronium graveolens Jacq. & 6 & 24 & 1,36 & 40 & 2,02 & 0,088 & 0,44 & 0,9 & 1,27 \\
\hline Nectandra saligna Nees & 5 & 20 & 1,14 & 40 & 2,02 & 0,123 & 0,62 & 0,88 & 1,26 \\
\hline Vernonanthura diffusa (Less.) H. Robinson & 5 & 20 & 1,14 & 40 & 2,02 & 0,101 & 0,51 & 0,82 & 1,22 \\
\hline Ceiba speciosa (A. St.-Hil.) Ravenna & 1 & 4 & 0,23 & 10 & 0,51 & 0,589 & 2,94 & 1,58 & 1,22 \\
\hline Cabralea canjerana (Vell.) Mart. & 6 & 24 & 1,36 & 30 & 1,52 & 0,117 & 0,58 & 0,97 & 1,15 \\
\hline Myrcia fallax DC. & 4 & 16 & 0,91 & 20 & 1,01 & 0,242 & 1,21 & 1,06 & 1,04 \\
\hline Machaerium nyctitans (Vell.) Benth. & 6 & 24 & 1,36 & 20 & 1,01 & 0,15 & 0,75 & 1,06 & 1,04 \\
\hline Pseudobombax grandiflorum (Cav.) A. Robyns. & 2 & 8 & 0,45 & 20 & 1,01 & 0,257 & 1,28 & 0,87 & 0,92 \\
\hline Trichilia palida Mart. & 4 & 16 & 0,91 & 30 & 1,52 & 0,037 & 0,19 & 0,55 & 0,87 \\
\hline Aegiphila sellowiana Cham. & 3 & 12 & 0,68 & 30 & 1,52 & 0,075 & 0,38 & 0,53 & 0,86 \\
\hline Eugenia sp. 1 & 3 & 12 & 0,68 & 30 & 1,52 & 0,041 & 0,21 & 0,44 & 0,8 \\
\hline Matayba elaeagnoides Radlk. & 3 & 12 & 0,68 & 30 & 1,52 & 0,028 & 0,14 & 0,41 & 0,78 \\
\hline Ocotea sp. & 3 & 12 & 0,68 & 30 & 1,52 & 0,027 & 0,13 & 0,41 & 0,78 \\
\hline Jacaranda puberula Cham. & 4 & 16 & 0,91 & 20 & 1,01 & 0,047 & 0,24 & 0,57 & 0,72 \\
\hline Casearia ulmifolia Vahl ex Vent. & 3 & 12 & 0,68 & 20 & 1,01 & 0,076 & 0,38 & 0,53 & 0,69 \\
\hline Xylopia sericea A. St. Hill & 2 & 8 & 0,45 & 20 & 1,01 & 0,11 & 0,55 & 0,5 & 0,67 \\
\hline Solanum leucodendron Sendtn. & 2 & 8 & 0,45 & 20 & 1,01 & 0,11 & 0,55 & 0,5 & 0,67 \\
\hline Bauhinia sp. & 3 & 12 & 0,68 & 20 & 1,01 & 0,062 & 0,31 & 0,5 & 0,67 \\
\hline Bathysa nicholsonii K. Schum. & 3 & 12 & 0,68 & 20 & 1,01 & 0,059 & 0,29 & 0,49 & 0,66 \\
\hline Cecropia hololeuca Miq. & 1 & 4 & 0,23 & 10 & 0,51 & 0,227 & 1,13 & 0,68 & 0,62 \\
\hline Mabea fistulifera Mart. & 2 & 8 & 0,45 & 20 & 1,01 & 0,07 & 0,35 & 0,4 & 0,6 \\
\hline Acacia polyphylla DC. & 2 & 8 & 0,45 & 20 & 1,01 & 0,037 & 0,18 & 0,32 & 0,55 \\
\hline Sorocea bonplandii (Baill.) & 2 & 8 & 0,45 & 20 & 1,01 & 0,036 & 0,18 & 0,32 & 0,55 \\
\hline Ouratea polygyna Engl. & 2 & 8 & 0,45 & 20 & 1,01 & 0,032 & 0,16 & 0,31 & 0,54 \\
\hline Guarea kunthiana A. Juss. & 3 & 12 & 0,68 & 10 & 0,51 & 0,085 & 0,42 & 0,55 & 0,54 \\
\hline
\end{tabular}


Tabela 1 - Cont.

Table 1 - Cont.

\begin{tabular}{|c|c|c|c|c|c|c|c|c|c|}
\hline Nectandra oppositifolia Nees. & 2 & $\overline{8}$ & $\overline{0,45}$ & 20 & $\overline{1,01}$ & $\overline{0,024}$ & 0,12 & 0,29 & $\overline{0,53}$ \\
\hline Sparattosperma leucanthum K. Schum. & 2 & 8 & 0,45 & 20 & 1,01 & 0,022 & 0,11 & 0,28 & 0,52 \\
\hline Casearia gossypiosperma Briq. & 3 & 12 & 0,68 & 10 & 0,51 & 0,072 & 0,36 & 0,52 & 0,52 \\
\hline Trichilia catigua A. Juss. & 2 & 8 & 0,45 & 20 & 1,01 & 0,019 & 0,09 & 0,27 & 0,52 \\
\hline Pseudopiptadenia contorta (DC.) & 3 & 12 & 0,68 & 10 & 0,51 & 0,038 & 0,19 & 0,44 & 0,46 \\
\hline Inga capitata Desv. & 1 & 4 & 0,23 & 10 & 0,51 & 0,057 & 0,28 & 0,26 & 0,34 \\
\hline Schizolobium parahyba (Vell.) Blake & 1 & 4 & 0,23 & 10 & 0,51 & 0,05 & 0,25 & 0,24 & 0,33 \\
\hline Erythrina verna Vell. & 1 & 4 & 0,23 & 10 & 0,51 & 0,039 & 0,19 & 0,21 & 0,31 \\
\hline Xylosma prockia (Turcz.) Turcz. & 1 & 4 & 0,23 & 10 & 0,51 & 0,036 & 0,18 & 0,2 & 0,3 \\
\hline Senna macranthera (Collad.) Irwin et Barn. & 1 & 4 & 0,23 & 10 & 0,51 & 0,032 & 0,16 & 0,19 & 0,3 \\
\hline Tapirira guianensis Aubl. & 1 & 4 & 0,23 & 10 & 0,51 & 0,029 & 0,14 & 0,19 & 0,29 \\
\hline Stryphnodendron guianense (Aubl.) Benth. & 1 & 4 & 0,23 & 10 & 0,51 & 0,022 & 0,11 & 0,17 & 0,28 \\
\hline Aloysia virgata (Ruiz \& Pavon) A. Juss. & 1 & 4 & 0,23 & 10 & 0,51 & 0,022 & 0,11 & 0,17 & 0,28 \\
\hline Solanum cernuum Vell. & 1 & 4 & 0,23 & 10 & 0,51 & 0,022 & 0,11 & 0,17 & 0,28 \\
\hline Platypodium elegans Vog. & 1 & 4 & 0,23 & 10 & 0,51 & 0,014 & 0,07 & 0,15 & 0,27 \\
\hline Guatteria sp. & 1 & 4 & 0,23 & 10 & 0,51 & 0,013 & 0,07 & 0,15 & 0,27 \\
\hline Amaioua guianensis Aubl. & 1 & 4 & 0,23 & 10 & 0,51 & 0,018 & 0,09 & 0,16 & 0,27 \\
\hline Guarea macropylla Vahl & 1 & 4 & 0,23 & 10 & 0,51 & 0,018 & 0,09 & 0,16 & 0,27 \\
\hline Croton floribundus Spreng. & 1 & 4 & 0,23 & 10 & 0,51 & 0,015 & 0,07 & 0,15 & 0,27 \\
\hline Platymiscium pubescens Micheli & 1 & 4 & 0,23 & 10 & 0,51 & 0,014 & 0,07 & 0,15 & 0,27 \\
\hline Erythroxylum pelleterianum A. St. Hil. & 1 & 4 & 0,23 & 10 & 0,51 & 0,012 & 0,06 & 0,14 & 0,26 \\
\hline Lacistema pubescens Mart. & 1 & 4 & 0,23 & 10 & 0,51 & 0,009 & 0,04 & 0,14 & 0,26 \\
\hline Aparisthmium cordatum (Juss.) Baill. & 1 & 4 & 0,23 & 10 & 0,51 & 0,01 & 0,05 & 0,14 & 0,26 \\
\hline Carpotroche brasiliensis Endl. & 1 & 4 & 0,23 & 10 & 0,51 & 0,008 & 0,04 & 0,13 & 0,26 \\
\hline Coffea arabica L. & 1 & 4 & 0,23 & 10 & 0,51 & 0,008 & 0,04 & 0,13 & 0,26 \\
\hline Coutarea hexandra (Jacq.) K. Schum. & 1 & 4 & 0,23 & 10 & 0,51 & 0,01 & 0,05 & 0,14 & 0,26 \\
\hline Miconia candolleana Triana & 1 & 4 & 0,23 & 10 & 0,51 & 0,011 & 0,06 & 0,14 & 0,26 \\
\hline Alchornea triplinervia (Spreng.) Müll.Arg. & 1 & 4 & 0,23 & 10 & 0,51 & 0,007 & 0,04 & 0,13 & 0,26 \\
\hline Trema micrantha Blume & 1 & 4 & 0,23 & 10 & 0,51 & 0,012 & 0,06 & 0,14 & 0,26 \\
\hline Cariniana estrellensis Kuntze & 1 & 4 & 0,23 & 10 & 0,51 & 0,008 & 0,04 & 0,13 & 0,26 \\
\hline Machaerium brasiliensis Vogel & 1 & 4 & 0,23 & 10 & 0,51 & 0,011 & 0,05 & 0,14 & 0,26 \\
\hline
\end{tabular}

Tabela 2 - Espécies arbóreas amostradas no trecho de floresta avançada da Mata da Agronomia, em Viçosa, MG, com os respectivos números de indivíduos (N) e parâmetros fitossociológicos: frequência absoluta (FA), frequência relativa (FR), densidade absoluta (DA), densidade relativa (DR), dominância absoluta (DoA), dominância relativa (DoR), valor de cobertura (VC) e valor de importância (VI), em ordem decrescente de VI.

Table 2 - Tree species sampled in advance forest in Mata da Agronomia, in Viçosa/MG, with their respective number of individuals $(N)$ and phytosociological parameters: absolute frequency (FA), relative frequency (FR), absolute density (DA), relative density (DR), absolute dominance (DoA), relative dominance (DoR), cover value (VC), importance value (VI\%), in decreased order of VI.

\begin{tabular}{|c|c|c|c|c|c|c|c|c|c|}
\hline Nome Científico & $\mathrm{N}$ & DA & DR & FA & FR & DoA & DoR & VC (\%) & VI $(\%)$ \\
\hline Anadenanthera peregrina Speg. & 28 & 112 & 7,37 & 90 & 4,5 & 6,09 & 26,52 & 16,95 & 12,8 \\
\hline Trichilia palida Mart. & 48 & 192 & 12,63 & 100 & 5 & 0,736 & 3,21 & 7,92 & 6,95 \\
\hline Morta & 23 & 92 & 6,05 & 90 & 4,5 & 1,595 & 6,95 & 6,5 & 5,83 \\
\hline Cariniana estrellensis Kuntze & 4 & 16 & 1,05 & 30 & 1,5 & 3,261 & 14,2 & 7,63 & 5,58 \\
\hline Piptadenia gonoacantha Macbride & 23 & 92 & 6,05 & 80 & 4 & 0,507 & 2,21 & 4,13 & 4,09 \\
\hline Rollinia silvatica Mart. & 17 & 68 & 4,47 & 80 & 4 & 0,825 & 3,59 & 4,03 & 4,02 \\
\hline Siparuna guianensis Aubl. & 20 & 80 & 5,26 & 90 & 4,5 & 0,309 & 1,35 & 3,3 & 3,7 \\
\hline Casearia gossypiosperma Briq. & 17 & 68 & 4,47 & 90 & 4,5 & 0,366 & 1,6 & 3,03 & 3,52 \\
\hline Dalbergia nigra Allem. ex Benth. & 17 & 68 & 4,47 & 80 & 4 & 0,364 & 1,59 & 3,03 & 3,35 \\
\hline
\end{tabular}

Continued... 
Tabela 2 - Cont.

Table 2 - Cont.

\begin{tabular}{|c|c|c|c|c|c|c|c|c|c|}
\hline Ceiba speciosa (A. St.-Hil.) Ravenna & 4 & 16 & 1,05 & 40 & 2 & 1,442 & 6,28 & 3,67 & 3,11 \\
\hline Platypodium elegans Vog. & 6 & 24 & 1,58 & 40 & 2 & 0,917 & 3,99 & 2,79 & 2,52 \\
\hline Luehea grandiflora Mart. \& Zucc. & 9 & 36 & 2,37 & 70 & 3,5 & 0,371 & 1,62 & 1,99 & 2,5 \\
\hline Terminalia argêntea Mart. & 15 & 60 & 3,95 & 40 & 2 & 0,301 & 1,31 & 2,63 & 2,42 \\
\hline Coutarea hexandra (Jacq.) K. Schum. & 12 & 48 & 3,16 & 30 & 1,5 & 0,387 & 1,69 & 2,42 & 2,11 \\
\hline Jacaranda puberula Cham. & 10 & 40 & 2,63 & 60 & 3 & 0,138 & 0,6 & 1,62 & 2,08 \\
\hline Casearia sp. & 9 & 36 & 2,37 & 50 & 2,5 & 0,288 & 1,26 & 1,81 & 2,04 \\
\hline Sparattosperma leucanthum K. Schum. & 4 & 16 & 1,05 & 30 & 1,5 & 0,799 & 3,48 & 2,27 & 2,01 \\
\hline Casearia sylvestris Sw. & 7 & 28 & 1,84 & 50 & 2,5 & 0,317 & 1,38 & 1,61 & 1,91 \\
\hline Protium warmingianum March. L. & 6 & 24 & 1,58 & 50 & 2,5 & 0,349 & 1,52 & 1,55 & 1,87 \\
\hline Amaioua guianensis Aubl. & 9 & 36 & 2,37 & 50 & 2,5 & 0,122 & 0,53 & 1,45 & 1,8 \\
\hline Apuleia leiocarpa Macbride & 7 & 28 & 1,84 & 50 & 2,5 & 0,221 & 0,96 & 1,4 & 1,77 \\
\hline Machaerium nyctitans (Vell.) Benth. & 3 & 12 & 0,79 & 30 & 1,5 & 0,557 & 2,43 & 1,61 & 1,57 \\
\hline Qualea jundiahy Warm. & 7 & 28 & 1,84 & 40 & 2 & 0,19 & 0,83 & 1,34 & 1,56 \\
\hline Sorocea bonplandii (Baill.) & 6 & 24 & 1,58 & 50 & 2,5 & 0,065 & 0,28 & 0,93 & 1,45 \\
\hline Zanthoxylum rhoifolium Lam. & 4 & 16 & 1,05 & 40 & 2 & 0,271 & 1,18 & 1,12 & 1,41 \\
\hline Syagrus romanzoffiana (Cham.) Glassm. & 3 & 12 & 0,79 & 30 & 1,5 & 0,405 & 1,76 & 1,28 & 1,35 \\
\hline Astronium graveolens Jacq. & 5 & 20 & 1,32 & 40 & 2 & 0,048 & 0,21 & 0,76 & 1,18 \\
\hline Copaifera langsdorffii Desf. & 5 & 20 & 1,32 & 30 & 1,5 & 0,105 & 0,46 & 0,89 & 1,09 \\
\hline Eugenia sp.1 & 5 & 20 & 1,32 & 30 & 1,5 & 0,086 & 0,37 & 0,84 & 1,06 \\
\hline Acacia polyphylla DC. & 4 & 16 & 1,05 & 30 & 1,5 & 0,145 & 0,63 & 0,84 & 1,06 \\
\hline Cupania vernalis Cambess. & 3 & 12 & 0,79 & 20 & 1 & 0,24 & 1,04 & 0,92 & 0,94 \\
\hline Maclura tinctoria (L.) D. Don ex Steud. & 3 & 12 & 0,79 & 30 & 1,5 & 0,087 & 0,38 & 0,58 & 0,89 \\
\hline Prunus sellowii Koehne & 3 & 12 & 0,79 & 30 & 1,5 & 0,03 & 0,13 & 0,46 & 0,81 \\
\hline Allophylus edulis Radlk. ex Warm. & 3 & 12 & 0,79 & 20 & 1 & 0,094 & 0,41 & 0,6 & 0,73 \\
\hline Matayba elaeagnoides Radlk. & 3 & 12 & 0,79 & 20 & 1 & 0,032 & 0,14 & 0,46 & 0,64 \\
\hline Maytenus aquifolia Mart. & 2 & 8 & 0,53 & 20 & 1 & 0,034 & 0,15 & 0,34 & 0,56 \\
\hline Albizia polycephala (Benth.) Killip & 2 & 8 & 0,53 & 20 & 1 & 0,031 & 0,14 & 0,33 & 0,55 \\
\hline Cariniana legalis (Mart.) Kuntze & 1 & 4 & 0,26 & 10 & 0,5 & 0,199 & 0,87 & 0,56 & 0,54 \\
\hline Cabralea canjerana (Vell.) Mart. & 1 & 4 & 0,26 & 10 & 0,5 & 0,163 & 0,71 & 0,49 & 0,49 \\
\hline Machaerium stipitatum Vog. & 1 & 4 & 0,26 & 10 & 0,5 & 0,105 & 0,46 & 0,36 & 0,41 \\
\hline Swartzia myrtifolia Smith & 2 & 8 & 0,53 & 10 & 0,5 & 0,025 & 0,11 & 0,32 & 0,38 \\
\hline Platymiscium pubescens Micheli & 1 & 4 & 0,26 & 10 & 0,5 & 0,044 & 0,19 & 0,23 & 0,32 \\
\hline Pseudobombax grandiflorum (Cav.) A. Robyns. & 1 & 4 & 0,26 & 10 & 0,5 & 0,041 & 0,18 & 0,22 & 0,31 \\
\hline Erythroxylum pelleterianum A. St. Hil. & 1 & 4 & 0,26 & 10 & 0,5 & 0,022 & 0,1 & 0,18 & 0,29 \\
\hline Icacinaceae 1 & 1 & 4 & 0,26 & 10 & 0,5 & 0,025 & 0,11 & 0,19 & 0,29 \\
\hline Eugenia sp. 2 & 1 & 4 & 0,26 & 10 & 0,5 & 0,022 & 0,1 & 0,18 & 0,29 \\
\hline Inga capitata Desv. & 1 & 4 & 0,26 & 10 & 0,5 & 0,022 & 0,09 & 0,18 & 0,29 \\
\hline Xylosma prockia (Turcz.) Turcz. & 1 & 4 & 0,26 & 10 & 0,5 & 0,018 & 0,08 & 0,17 & 0,28 \\
\hline Myrtaceae 1 & 1 & 4 & 0,26 & 10 & 0,5 & 0,016 & 0,07 & 0,17 & 0,28 \\
\hline Guettarda viburnoides Cham. \& Schltdl. & 1 & 4 & 0,26 & 10 & 0,5 & 0,018 & 0,08 & 0,17 & 0,28 \\
\hline Eriotheca candolleana (K. Schum.) A. Rob. & 1 & 4 & 0,26 & 10 & 0,5 & 0,018 & 0,08 & 0,17 & 0,28 \\
\hline Guapira opposita (Vell.) Reitz & 1 & 4 & 0,26 & 10 & 0,5 & 0,016 & 0,07 & 0,17 & 0,28 \\
\hline Senna multijuga (Rich.) Irwin et Barn. & 1 & 4 & 0,26 & 10 & 0,5 & 0,016 & 0,07 & 0,17 & 0,28 \\
\hline Solanum cernuum Vell. & 1 & 4 & 0,26 & 10 & 0,5 & 0,013 & 0,06 & 0,16 & 0,27 \\
\hline Stryphnodendron guianense (Aubl.) Benth.) & 1 & 4 & 0,26 & 10 & 0,5 & 0,008 & 0,04 & 0,15 & 0,27 \\
\hline Mabea fistulifera Mart. & 1 & 4 & 0,26 & 10 & 0,5 & 0,009 & 0,04 & 0,15 & 0,27 \\
\hline Nectandra oppositifolia Nees. & 1 & 4 & 0,26 & 10 & 0,5 & 0,01 & 0,04 & 0,15 & 0,27 \\
\hline Brunfelsia uniflora (Pohl) D. Don & 1 & 4 & 0,26 & 10 & 0,5 & 0,012 & 0,05 & 0,16 & 0,27 \\
\hline Myrcia fallax DC. & 1 & 4 & 0,26 & 10 & 0,5 & 0,008 & 0,03 & 0,15 & 0,27 \\
\hline Pseudopiptadenia contorta (DC.) & 1 & 4 & 0,26 & 10 & 0,5 & 0,007 & 0,03 & 0,15 & 0,26 \\
\hline
\end{tabular}

Revista Árvore, Viçosa-MG, v.35, n.3, p.493-503, 2011 
A família Fabaceae apresentou o primeiro lugar na ordenação de VI (28,7\%), fato explicado pela alta densidade da população de Anadenanthera peregrina, Piptadenia gonoacantha e Apuleia leiocarpa, que juntas corresponderam a 71,5\% dos seus indivíduos. Além disso, as cinco primeiras famílias com maior valor de importância nesse primeiro trecho somaram mais de 58\% desse índice de valoração, classificando essas famílias como as representativas da florística da floresta inicial (Figura 1).

As famílias que apresentaram maior número de espécies na floresta avançada foram Fabaceae (16), Salicaceae (4) e Malvaceae (4), sobressaindo Fabaceae (103), Meliaceae (49) e Flacourtiaceae (34) com o maior número de indivíduos. Semelhante ao observado na floresta inicial, a família Fabaceae foi a que apresentou o maior valor de importância (VI = 30,5\%), sendo $A$. peregrina, $P$. gonoacantha e Dalbergia nigra as espécies que mais contribuíram para esse valor, devido à alta densidade, concentrando 66,0\% dos indivíduos amostrados. As cinco primeiras famílias, do total das 26 amostradas, obtiveram 55,7\% do VI nesse trecho de floresta (Figura 1).

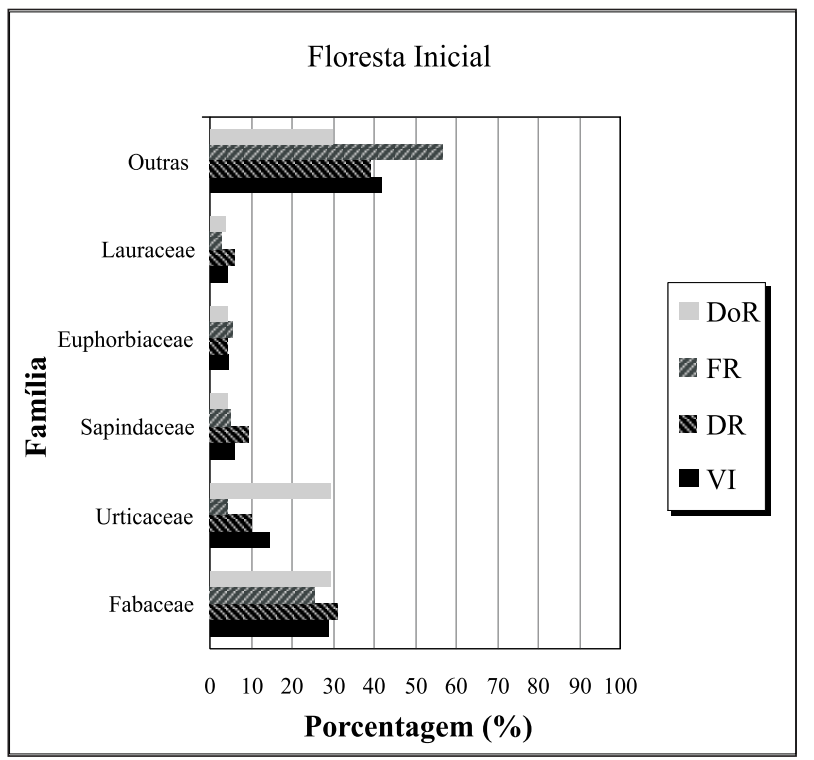

Considerando os dois trechos como um contínuo, as 10 famílias que apresentaram maior VI em ordem decrescente foram: Fabaceae (30,0\%), Urticaceae (7,0\%), Salicaceae (5,4\%), Meliaceae (5,3\%), Malvaceae (5,2\%), Annonaceae (4,8\%), Sapindaceae (4,3\%), Morta (4,2\%), Monimiaceae (3,5\%) e Lecythidaceae (3,4\%).

Com relação aos índices de diversidade de Shannon (H') e à equabilidade ( $\mathrm{J}$ '), observou-se que a diversidade específica $\left(H^{\prime}=3,57\right)$ da floresta inicial não apresentou muita variação quando comparada com a floresta avançada $\left(H^{\prime}=3,49\right)$. A equabilidade observada para a florestal inicial foi de J' = 0,82 e de J' = 0,85. No contínuo, o índice de Shannon ( $\mathrm{H}^{\prime}$ ) foi de 3,82 nat.ind. ${ }^{-1}$ e a equabilidade (J'), 0,84.

A classificação sucessional das espécies amostradas nos dois trechos de floresta analisados é demonstrada na Figura 2. Os gráficos comparativos evidenciam a substituição progressiva dos indivíduos de espécies pioneiras e secundárias iniciais, em quantidade superior na floresta inicial, por indivíduos de espécies classificadas como secundárias, que aumentam no trecho de floresta avançada.

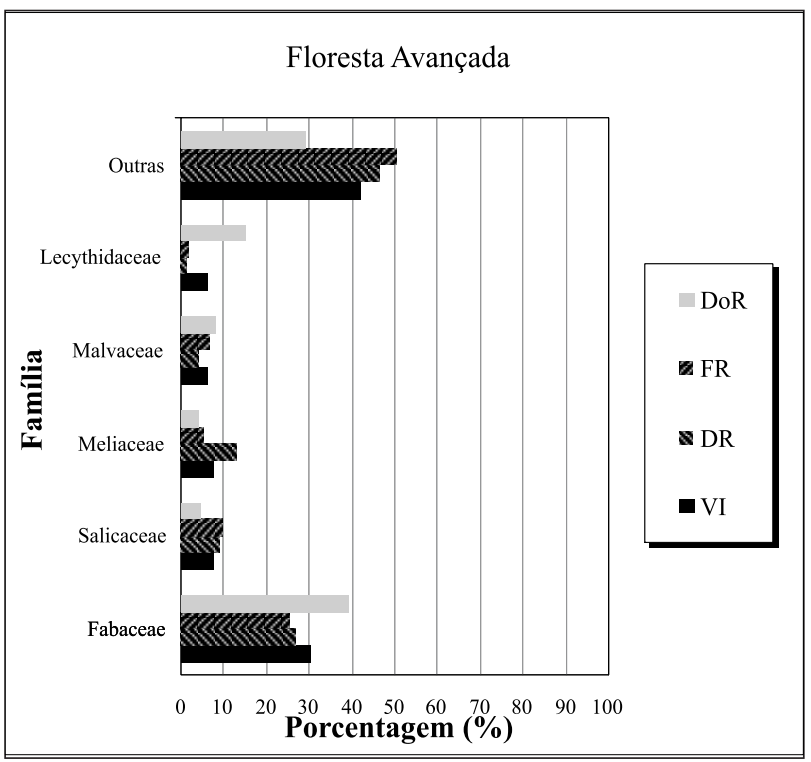

Figura 1 - Parâmetros fitossociológicos (FR = frequência relativa; DR = densidade relativa; DoR = dominância relativa) das cinco famílias de maior valor de importância (VI) amostradas na floresta inicial e na floresta avançada da Mata da Agronomia, em Viçosa, MG, Brasil.

Figure 1 - Phytosociological parameters $(F R=$ relative frequence; $D R=$ relative density; DoR = relative dominance; VI= importance value) of the five families presenting the highest value of importance (VI) sampled in initial forest and advance forest in Mata da Agronomia in Viçosa, MG, Brasil. 

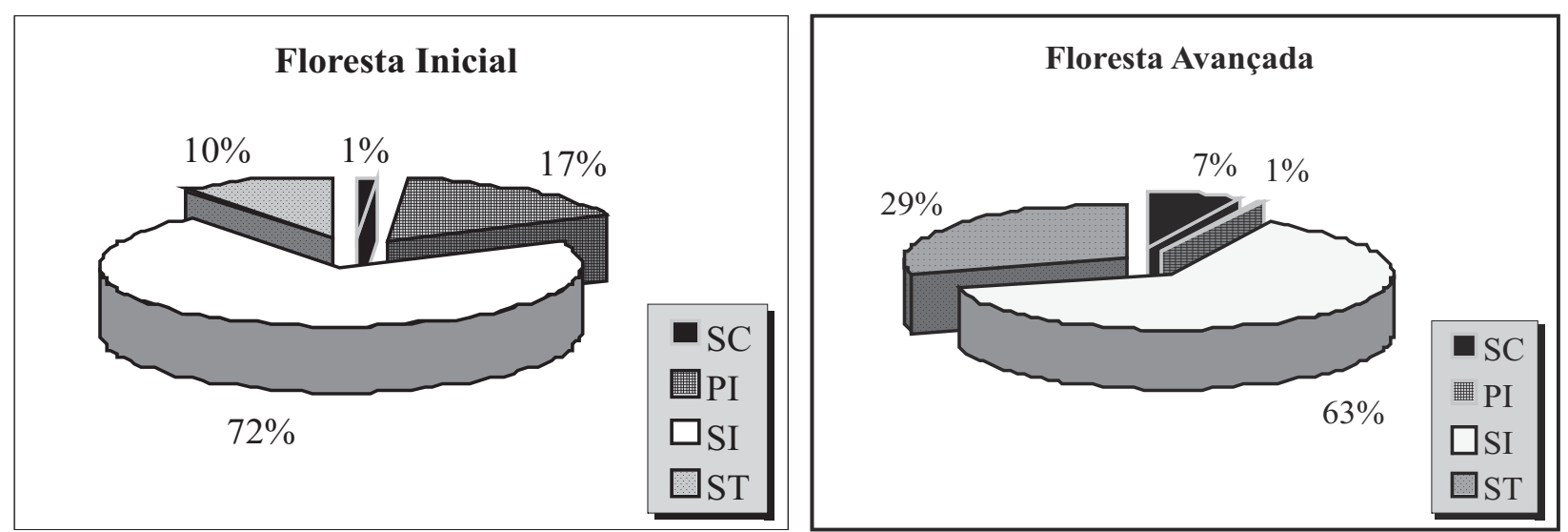

Figura 2 - Percentual de espécies por categoria sucessional (SC = sem classificação, PI = pioneira, SI = secundária inicial e ST = secundária tardia) amostradas em cada trecho de floresta da Mata da Agronomia, em Viçosa, MG, Brasil.

Figure 2 - Percentage of species per sucessional categories $(S C=$ no classification; $P I=$ pioneer, $S I=$ initial secondary e ST = late secondary) sampled in each site of Forest in Mata da Agronomia in Viçosa, MG, Brazil.

Em relação aos solos das florestas inicial e avançada, esses se diferenciaram quanto à classe textural, sendo classificadas como Argilosa e ArgiloArenosa, respectivamente, apresentando diferenças significativas em relação ao teor de areia. Os maiores teores de macronutrientes ( $\mathrm{P}, \mathrm{K}$, Ca e Mg) foram encontrados na floresta inicial, assim como os menores teores de alumínio (Al), que resultaram em valores superiores da soma de base (SB) e da saturação por base (V) e elevou o pH. Os resultados apontaram o solo da floresta avançada como o mais ácido, distrófico, sendo os teores de todos os macronutrientes menores que os do solo da floresta inicial (Tabela 3).

\section{DISCUSSÃO}

O total de espécies identificadas neste estudo acompanha os resultados encontrados nos levantamentos realizados em Florestas Estacionais Semideciduais da região de Viçosa, Minas Gerais, onde 91 espécies foram amostradas por Silva et al. (2000), 94 espécies por Paula et al. (2002) e 107 espécies por Ribas et al. (2003), em dois trechos de florestas. Valores superiores foram obtidos por Silva et al. (2004), que encontraram 124 espécies; e por Campos et al. (2006), que identificaram 151. Os valores encontrados neste estudo, segundo Ribas et al. (2003), podem ser considerados expressivos em relação à maioria dos trabalhos realizados nesse mesmo tipo de formação florestal da Zona da Mata mineira.
Os resultados demonstram o avanço do estádio de sucessão na floresta inicial, em meio às espécies típicas de início de sucessão, como Trema micrantha, Mabea fistulifera, Cecropia glaziovii, C. hololeuca, Croton urucurana, Aegiphila sellowiana, Solanum leucodendron e Vernonanthura diffusa, entre outras. Foi constatado o estabelecimento de espécies características de estádios sucessionais mais avançados, como Copaifera langsdorffii, Cabralea canjerana, Cariniana estrellensis, Dalbergia nigra, Trichilia palida e Zanthoxylum rhoifolium, amostradas na floresta avançada.

A Dalbergia nigra, com 21 indivíduos nas duas áreas, merece destaque, pois figura na Portaria $\mathrm{n}^{\circ}$. 37-N, de 03/04/92 do IBAMA, como espécie ameaçada de extinção, destacando-se ainda a presença de grandes indivíduos de C. legalis e C. estrellensis, infrequentes na região por conta da qualidade da sua madeira de grande valor comercial (SILVA et al., 2004), o que justifica a preservação do fragmento.

A família Fabaceae destacou-se em número de espécies também em outros trabalhos realizados em Floresta Estacional Semidecidual na região de Viçosa, MG (MARANGON et al., 2003; RIBAS et al., 2003; SILVA et al., 2004; PINTO et al., 2007; FERREIRA JÚNIOR et al., 2007). Segundo Silva et al. (2004), a alta riqueza apresentada por essa família botânica na região pode estar relacionada à capacidade simbiótica de fixar nitrogênio apresentada por muitas das espécies que a compõem, o que as capacitam a se estabelecer em solos com baixa fertilidade natural, muito presentes nas encostas e topos de morros. 
Tabela 3 - Resultado das análises químicas e físicas de 20 amostras de solo superficial (camada de 0-10 cm) coletadas nos trechos de florestas inicial e avançada na Mata da Agronomia, em Viçosa, MG, Brasil.

Table 3 - Results of chemical and physical analyses of 20 shallow soil samples $(0-10 \mathrm{~cm}$ layer) collected in the initial and advance forest in Mata da Agronomia, in Viçosa, MG, Brazil.

\begin{tabular}{|c|c|c|c|}
\hline & $\begin{array}{l}\text { Floresta Inicial } \\
\text { Média } \pm \text { Desvio }\end{array}$ & $\begin{array}{c}\text { Floresta Avançada } \\
\text { Média } \pm \text { Desvio }\end{array}$ & $\mathrm{p}^{1}$ \\
\hline $\mathrm{pH}$ em $\mathrm{H}_{2} \mathrm{O}$ & $5,71 \pm 0,95$ & $4,91 \pm 0,36$ & $0,024 * *$ \\
\hline $\mathrm{P}\left(\mathrm{mg} \cdot \mathrm{dm}^{-3}\right)$ & $1,32 \pm 1,13$ & $1,16 \pm 0,93$ & $0,733^{\mathrm{NS}}$ \\
\hline $\mathrm{K}\left(\mathrm{mg} \cdot \mathrm{dm}^{-3}\right)$ & $95,00 \pm 42,53$ & $77,50 \pm 19,58$ & $0,253^{\mathrm{NS}}$ \\
\hline $\mathrm{Ca}\left(\mathrm{cmol}_{\mathrm{c}} \cdot \mathrm{dm}^{-3}\right)$ & $4,66 \pm 2,60$ & $1,99 \pm 1,01$ & $0,007 * *$ \\
\hline $\operatorname{Mg}\left(\mathrm{cmolc}^{\mathrm{c}} \cdot \mathrm{dm}^{-3}\right)$ & $1,64 \pm 0,86$ & $1,14 \pm 0,27$ & $0,093^{\mathrm{NS}}$ \\
\hline $\mathrm{Al}\left(\mathrm{cmol}_{\mathrm{c}} \cdot \mathrm{dm}^{-3}\right)$ & $0,33 \pm 0,63$ & $0,39 \pm 0,35$ & $0,878^{\mathrm{NS}}$ \\
\hline $\mathrm{H}+\mathrm{Al}\left(\mathrm{cmol}_{\mathrm{c}} \cdot \mathrm{dm}^{-3}\right)$ & $5,05 \pm 3,09$ & $8,32 \pm 1,78$ & $0,009 * *$ \\
\hline $\mathrm{SB}\left(\mathrm{cmol}_{\mathrm{c}} \cdot \mathrm{dm}^{-3}\right)^{2}$ & $6,54 \pm 3,52$ & $4,11 \pm 0,94$ & $0,048 * *$ \\
\hline $\mathrm{t}\left(\mathrm{cmol}_{\mathrm{c}} \cdot \mathrm{dm}^{-3}\right)^{2}$ & $6,87 \pm 2,97$ & $3,70 \pm 1,02$ & $0,005 * *$ \\
\hline $\mathrm{T}\left(\mathrm{cmol}_{\mathrm{c}}^{\mathrm{c}} \cdot \mathrm{dm}^{-3}\right)^{2}$ & $11,59 \pm 1,10$ & $11,65 \pm 1,30$ & $0,921^{\mathrm{NS}}$ \\
\hline $\mathrm{V}(\%)^{2}$ & $55,54 \pm 28,32$ & $28,78 \pm 11,80$ & $0,012 * *$ \\
\hline $\mathrm{m}(\%)^{2}$ & $13,32 \pm 26,38$ & $12,45 \pm 12,23$ & $0,925^{\mathrm{NS}}$ \\
\hline $\mathrm{MO}\left(\mathrm{dag} \cdot \mathrm{kg}^{-1}\right)^{2}$ & $5,65 \pm 0,73$ & $6,26 \pm 0,61$ & $0,058^{\mathrm{NS}}$ \\
\hline P-rem $\left(\mathrm{mg} \cdot \mathrm{L}^{-1}\right)^{2}$ & $33,94 \pm 6,02$ & $27,78 \pm 5,21$ & $0,024 * *$ \\
\hline Areia (\%) & $40,10 \pm 5,02$ & $45,70 \pm 3,77$ & $0,011 * *$ \\
\hline Argila (\%) & $15,29 \pm 6,30$ & $12,80 \pm 2,89$ & $0,288^{\mathrm{NS}}$ \\
\hline Silte (\%) & $44,70 \pm 8,96$ & $41,50 \pm 4,71$ & $0,338^{\mathrm{NS}}$ \\
\hline
\end{tabular}

${ }^{1}$ As comparações foram realizadas pelo teste t de Student $(n=10)$, em que : **significativo a $5 \%$; ${ }^{\text {Ns }}$ não significante; ${ }^{2}$ SB $=$ soma de base trocáveis; $\mathrm{t}$ = capacidade de troca catiônica efetiva; $\mathrm{T}$ = capacidade de troca catiônica a pH 7,0; $\mathrm{V}=$ índice de saturação por base; $\mathrm{m}=$ índice de saturação por alumínio; MO = matéria orgânica; e P-rem = fósforo remanescente.

Observou-se que a diversidade específica da floresta inicial não apresentou muita variação quando comparada com a da floresta avançada. Os valores estimados de Shannon (H') são compatíveis com os valores de referência propostos para Florestas Semideciduais da região, que variam entre 3,2 e 4,2 (MEIRA-NETO; MARTINS, 2000). O valor obtido para a equabilidade (J') indica heterogeneidade florística alta para o componente arbóreo.

A floresta inicial abriga indivíduos de espécies pioneiras típicas, muito comuns ao estádio de sucessão em que esse trecho de floresta se encontra e de ampla distribuição pelas Florestas Estacionais Semideciduais da Região Sudeste do país, como C. hololeuca, C. glaziovii, Croton urucurana, Trema micrantha e Vernonanthura diffusa, entre outras. A floresta avançada abriga grande concentração de indivíduos de Cariniana estrellensis e Cariniana legalis, raros na região, em decorrência do grande valor comercial da sua madeira (SILVA et al., 2004), além de indivíduos de Qualea jundiahy, Swartzia myrtifolia e Trichilia palida, entre outras, que justificam a classificação desse trecho como de floresta secundária avançada.
Assim, de acordo com o resultado apresentado nos gráficos, tendo como parâmetro a RESOLUÇÃO CONAMA n ${ }^{\circ} 10$, de $1^{\circ}$ de outubro de 1993 (publicada no DOU n ${ }^{\circ}$. 209, de 3 de novembro de 1993, Seção 1 , páginas 16497-16498), a floresta inicial encontra-se em fase de transição, do estádio inicial para o médio, enquanto o estádio de sucessão do segundo trecho de floresta se enquadra como o de floresta avançada, conforme os parâmetros básicos descritos nessa Resolução para vegetação de Mata Atlântica.

Comprova-se, contudo, que cada um dos dois trechos apresentou distinta identidade florística e estrutural. Essa diferença entre os trechos tende a se estreitar com o aumento do sombreamento na floresta inicial, sendo esse fundamental na dinâmica da comunidade para o recrutamento de espécies secundárias tardias e o avanço da sucessão.

Os resultados apresentados servem de parâmetro para novos estudos de dinâmica na área e permitem recomendar estratégias para programas de manejo, preservação e recuperação ambiental.

No que tange ao solo, a floresta inicial está inserida em solo eutrófico com bons teores de nutrientes. Esperava-se o contrário, ou seja, no estádio mais 
avançado maior fertilidade do solo devido ao maior aporte de nutrientes resultante da ciclagem da serapilheira depositada, como foi constatado em outra floresta da região de Viçosa (PINTO et al., 2007). Todavia, naquele estudo o trecho mais avançado de sucessão estava localizado em terrenos de baixada com solo naturalmente mais fértil, ao passo que o trecho inicial se encontrava sobre solos de encostas, naturalmente mais pobres em virtude da lixiviação de nutrientes.

As características do solo influenciam o processo de sucessão nas florestas, podendo acelerá-lo nos ambientes com maior fertilidade (PINTO et al., 2007), como pode ser observado principalmente na floresta inicial tanto no aspecto sucessional com recrutamento de novas espécies tolerantes à sombra quanto no porte das espécies pioneiras amostradas na comunidade.

A comparação dos resultados desses dois estudos sugere que a posição topográfica e o solo associado a ela exercem maior influência na fertilidade do solo que o estádio sucessional da floresta.

\section{AGRADECIMENTOS}

Ao Conselho Nacional de Desenvolvimento Científico e Tecnológico (CNPq) pelo auxílio a esta pesquisa e a bolsa concedida.

\section{REFERÊNCIAS}

APG II. ANGIOSPERM PHYLOGENY GROUP II. An update of the Angiosperm Phylogeny Group classification for the orders and families of flowering plant: APG II. Botanical Journal of the Linnaean Society, v. 141, n. 4, p. 399-436, 2003.

BROWER, J. E.; ZAR, J. H. Field and laboratory methods for general ecology. 2. ed. Ames: Wm. C. Brown Company, 1984. 226 p.

CAMPOS, E. P.et al. Florística e estrutura horizontal da vegetação arbórea de uma ravina em um fragmento florestal no município de Viçosa, MG. Revista Árvore, v. 30, n. 6, p. 1045-1054, 2006.

CASTRO, P. S.et al. Interceptação da chuva por mata natural secundária na região de Viçosa, MG. Revista Árvore, v. 7, p. 76-79, 1983.

CIEnTEC. Mata Nativa 2: Manual do Usuário. Viçosa, MG: Cientec, 2006. 295 p.

Revista Árvore, Viçosa-MG, v.35, n.3, p.493-503, 2011
COSTA, C.M.R. et al. (Org.). Biodiversidade em Minas Gerais: um Atlas para a conservação. Belo Horizonte: Fundação Biodiversitas, 1998. 92p.

FERREIRA JÚNIOR, W.G. et al.Composição florística da vegetação arbórea de um trecho de floresta estacional semidecídua em Viçosa, Minas Gerais, e espécies de maior ocorrência na região. Revista Árvore, v. 31, n. 6, p. 1121-1130, 2007.

GANDOLFI, S.; LEITÃO FILHO, H. F.; BEZERRA, C. L. F. Levantamento florístico e caráter sucessional das espécies arbustivo-arbóreas de uma floresta mesófila semidecídua no município de Guarulhos, SP. Revista Brasileira de Biologia, v. 55, n. 4, p. 753-767, 1995.

MARANGON, L.; SOARES, J. J.; FELICIANO, A. L. P. Florística arbórea da Mata da Pedreira, município de Viçosa, Minas Gerais. Revista Árvore, v. 27, n. 2, p. 207-215, 2003.

MARTINS, S.V.; PELLEGRINI, M.C.; MARANGON, L.C. Composição florística e estrutura de uma floresta secundária no município de Cruzeiro-SP. Revista Árvore, v. 26, n. 1, p. 35-41, 2002.

MARTINS, S. V.; RODRIGUES, R.R. Gap-phase regeneration in a semideciduous mesophytic forest, south-eastern Brazil. Plant Ecology, v. 163, p. 51-62, 2002.

MARTINS, S. V. et al. Colonization of gaps produced by death of bamboo clumps in a semideciduous mesophytic forest in south-eastern Brazil. Plant Ecology, v. 172, n. 1, p. 121-131, 2004.

MEIRA NETO, J. A. A.; SILVA, A. E. Caracterização dos fragmentos florestais das áreas de influencia e diretamente afetada pela UHE de Pilar, Vale do Piranga, Zona da Mata de Minas Gerais. Viçosa, MG: FUNARBE, Universidade Federal de Viçosa, 1995. 57p.

MEIRA NETO, J.A. A.; MARTINS, F. Estrutura da Mata da Silvicultura, uma Floresta Estacional Semidecidual Montana no município de Viçosa-MG. Revista Árvore, v. 24, n. 2, p. 151-160, 2000.

MUELLER-DOMBOIS, D.; ELLENBERG, H. Aims and methods of vegetation ecology. New York: John Wiley \& Sons, 1974. 547 p. 
OLIVEIRA-FILHO, A. T. et al. Estrutura fitossociológica e variáveis ambientais em um trecho de mata ciliar do córrego dos Vilas Boas, Reserva Biológica do Poço Bonito, Lavras (MG). Revista Brasileira de Botânica, v. 17, n. 1, p. 67-85, 1994.

PAULA, A. et al. Alterações florísticas ocorridas num período de quatorze anos na vegetação arbórea de uma floresta estacional semidecidual em Viçosa-MG. Revista Árvore, v. 26, n. 6, p. 743-749, 2002.

PINTO, S. I. C. Florística, estrutura e ciclagem de nutrientes em dois trechos de Floresta Estacional Semidecidual na Reserva Florestal Mata do Paraíso, Viçosa-MG. 2005. 110 f. Dissertação (Mestrado em Ciência Florestal) - Universidade Federal de Viçosa, Viçosa, MG, 2005.

PINTO, S. I. C. et al. Estrutura do componente arbustivo-arbóreo de dois estádios sucessionais de Floresta Estacional Semidecidual na reserva florestal Mata do Paraíso, Viçosa, MG, Brasil. Revista Árvore, v. 31, n. 5, p. 823-833, 2007.
RESENDE, M.; CURI, N.; SANTANA, D. P. Pedologia e fertilidade do solo: interações e interpretações. Brasília: Ministério da Agricultura; Lavras: Ufla/Faep, 1988. 83 p.

RIBAS, R.F. et al. Composição florística de dois trechos em diferentes etapas serais de uma floresta estacional semidecidual em Viçosa, Minas Gerais. Revista Árvore, v. 27, n. 6, p. 821-830, 2003.

SILVA, A. F.; FONTES, N. R. L.; LEITÃO-FILHO, H. F. Composição florística e estrutura horizontal do estrato arbóreo de um trecho da Mata da Biologia da Universidade Federal de Viçosa Zona da Mata de Minas Gerais. Revista Árvore, v. 24, n. 4, p. 397-405, 2000.

SILVA, N. R. S.; MARTINS, S. V.; MEIRA NETO, J. A. A. Composição florística e estrutura de uma Floresta Estacional Semidecidual Montana em Viçosa, MG. Revista Árvore, v. 28, n. 3, p. 397-405, 2004.

VELOSO, H. P.; RANGEL-FILHO, A. L. R.; LIMA, J. C. A. Classificação da vegetação brasileira adaptada a um sistema universal. Rio de Janeiro: IBGE, 1991. 123 p. 